\title{
Knowledge and Technology Transfer in KM3NeT
}

\author{
Ekaterini Tzamariudaki ${ }^{1, *}$ and Konstantinos Pikounis ${ }^{1,2}$ \\ on behalf of the KM3NeT Collaboration \\ ${ }^{1}$ Institute of Nuclear and Particle Physics, N.C.S.R. Demokritos, Patriarchou Grigoriou and \\ Neapoleos 27, Agia Paraskevi, Athens, Greece \\ ${ }^{2}$ National Technical University of Athens, Heroon Polytexneiou 9, Zografou Campus, Athens, Greece
}

\begin{abstract}
The status of the construction of the KM3NeT detector components and the experience gained from the detector units deployed underpin the dissemination of innovative technological solutions to industry, Institutions and other potentially interested parties. KM3NeT is now entering a phase during which the design of the detector elements is being finalized and testing procedures for controlling the quality of the different components are settled. In addition, a few KM3NeT detection units have been deployed successfully and many more are coming, data have been recorded and are currently being analysed. The maturity and scalability of the technological solutions developed or modified by $\mathrm{KM} 3 \mathrm{NeT}$ has been demonstrated and therefore justifies their exposure to relevant technology developers or users.
\end{abstract}

\section{Introduction}

$\mathrm{KM} 3 \mathrm{NeT}$ is a large research infrastructure currently under construction. When completed, it will consist of a network of deep-sea detectors with user ports for Earth and Sea sciences, deployed at the Mediterranean sea [1]. KM3NeT/ARCA (Astroparticle Research with Cosmics in the Abyss), aims at the discovery and subsequent observation of high-energy neutrino sources in the Universe and is currently under construction at a depth of $3500 \mathrm{~m}$, about $80 \mathrm{~km}$ off-shore Portopalo di Capo Passero in Sicily. KM3NeT/ORCA (Oscillation Research with Cosmics in the Abyss) at a depth of $2450 \mathrm{~m}$, about $40 \mathrm{~km}$ offshore from Toulon, will use atmospheric neutrino oscillations at low energies to measure neutrino fundamental properties focusing on the neutrino mass ordering. During the design phase and as construction is progressing, it has often been the case that KM3NeT needs have motivated developments in technology. Although the broad scientific program of KM3NeT focuses on fundamental research, knowledge and technology transfer are highly valued as a way to strengthen the excellence of the collaborating institutes, improve collaboration with the leaders of technological advancements, train the future scientists and most importantly, as return to society. In this article the knowledge and technology transfer activities in $\mathrm{KM} 3 \mathrm{NeT}$ are presented.

\footnotetext{
* Corresponding author: katerina@inp.demokritos.gr
} 


\section{Knowledge and Technology Transfer}

Knowledge and technology transfer concerns the dissemination of technical know-how and of technological innovations. At this stage the KM3NeT design phase has been concluded and construction is progressing. Few detection units have been deployed successfully both in Italy and France and first results for the depth dependence of the atmospheric muon rate, using the data recorded, have been presented. Currently, KM3NeT has the potential to contribute with technological solutions and expertise in the fields of deep-sea technology and operations, optical and acoustic measurements, low power electronics, communication technologies and computing and data sciences. The strategy followed for knowledge and technology transfer focuses on: i) maximising the flow of information between KM3NeT and the developers of technological advances, ii) exposing developed or adapted technological choices and innovative solutions to interested parties in other Institutions or in industry and iii) technology transfer of services developed by KM3NeT.

The following technological solutions developed by KM3NeT could be exploited by other Institutes or by industry:

- Tool for connecting and disconnecting wet-mateable connectors with light ROVs

The detection units are connected to the seabed network via electro-optical wetmateable connectors. These typically require at least $60 \mathrm{~kg}$ of force for (dis)connection, which can only be applied by a heavy duty ROV. CPPM (Centre de Physique des Particules de Marseille) has developed a tool to connect wet-mateable connectors which decreases the maximum force necessary for the ROV to deliver to less than $30 \mathrm{~kg}$. Consequently, KM3NeT sea operations in France can use a lightweight ROV, which is less expensive and has better availability.

- Multi-PMT testing facility [2]

A "DarkBox" system has been developed by INFN (Instituto Nationale di Fisica Nucleare) Napoli, which is capable of measuring the characteristics of 62 Photomultipliers (PMTs) simultaneously twice a day. The design allows easy, fast and safe loading and unloading of the PMTs, thus optimizing the operation time. The PMTs are maintained in a vertical position using PVC collars and elastic bands. Dark tightness is achieved by means of light-tight supports for each PMT. It can be used to measure the PMT dark rate, equalize gain, determine spurious pulses and timing characteristics such as transit time and transit time spread. A complete PMT characterisation cycle takes about 10 hours of which 9 hours are used for PMT conditioning in darkness.

- Precision in-water positioning [3-5]

During the deployment phase, the absolute positioning of the mechanical structures on the seafloor is provided by commercial instrumentation that allows a precision of about $2 \mathrm{~m}$. For the needs of the experiment, INFN developed a relative acoustic positioning system (RAPS) for precise positioning of the floating optical modules along the detection units. RAPS is formed by a Long Baseline (LBL) of acoustic transmitters (beacons) and receivers (hydrophones) placed on the seafloor in fixed positions and acoustic sensors mounted in the optical modules to measure the 
displacement of the DUs under the effect of sea-currents. Acoustic receivers and transmitters are time-synchronised via absolute GPS time distributed from shore. RAPS is designed to achieve a position accuracy of $10 \mathrm{~cm}$.

- Data acquisition [6]

- Lightweight Web server library

Detector monitoring and management require the transmission of data to and from the KM3NeT detector components which are located at large sea depths. KM3NeT uses in-house made Web server libraries to simplify the development of Graphical User Interfaces for the Detector and Acquisition Control system (Control Unit) and for the central Data Base Web Application Server. The HTTP(S) interface is a thin library $(36 \mathrm{KiB})$ that can be incorporated also into existing applications. While the library is feature-rich, the simplicity of code and the lack-by-design of dangerous functions (e.g. page upload) reduces the attack surface and simplifies secure application development.

- SAWI: Server Application with Web Interface

The software that controls detector operation and data acquisition in KM3NeT (Control Unit) runs as separate processes possibly hosted on different machines and interconnected by a remote procedure call network protocol. Given the availability of in-house Web access instead of using a different messaging protocol, KM3NeT developed its own remote procedure call technique, named Server Application with Web Interface (SAWI) that uses HTTP(S) as transport protocol. The SAWI library is extremely lightweight (less than $20 \mathrm{KiB}$ ) and suitable for usage under Mono or .NET/.NET Core (Linux, Windows, MacOS).

\section{Conclusions}

As construction of the $\mathrm{KM} 3 \mathrm{NeT}$ detectors is progressing, the Collaboration intends to maximise both the scientific outcome of the project and the return to society. Knowledge transfer and technology dissemination is pursued by establishing a two-way flow of technological know-how and innovation between KM3NeT and industry, as well as between KM3NeT and other Institutions. Technology transfer in the form of services can also be provided by $\mathrm{KM} 3 \mathrm{NeT}$ to industry or to Institutions with potential interest.

\section{References}

1. Adrian-Martinez et al. (KM3NeT Coll.), J. Phys. G43 084001 (2016)

2. C. M. Mollo et al. (KM3NeT Coll.), EPJ Web Conf. 11606010 (2016)

3. S. Viola, R. Coniglione et al. (KM3NeT Coll.), PoS ICRC2017 1031 (2018)

4. S. Viola, F. Simeone, M. Saldana et al. (KM3NeT Coll.), EPJ Web Conf. 11103006 (2016)

5. G. Riccobene et al. (KM3NeT Coll.), Position calibration with KM3NeT, in these proceedings

6. C. Bozza et al. (KM3NeT Coll.), KM3NeT Acquisition Control, in these proceedings 